\title{
Impact of Fast-Acting Insulin Aspart on Glycemic Control in Patients with Type 1 Diabetes Using Intermittent-Scanning Continuous Glucose Monitoring Within a Real-World Setting: The GoBolus Study
}

\author{
Thomas Danne, MD, Matthias Axel Schweitzer, MD, ${ }^{2}$ Winfried Keuthage, $\mathrm{MD}^{3}$ Stefan Kipper, MS, ${ }^{2}$ \\ Yasmin Kretzschmar, MD, Jörg Simon, MD, Tanja Wiedenmann, ${ }^{4} D_{1}^{2}$ and Ralph Ziegler, $\mathrm{MD}^{5}$
}

\begin{abstract}
Background: The GoBolus study investigated the real-world effectiveness of faster aspart in patients with type 1 diabetes (T1D) using intermittent-scanning continuous glucose monitoring (iscCGM) systems.

Methods: This 24-week, multicenter, single-arm, noninterventional study investigated adults with T1D ( $\mathrm{HbA}_{1 \mathrm{c}}$, $7.5 \%-9.5 \%$ ) receiving multiple daily injections (MDI) of insulin and using iscCGM within local healthcare settings for $\geq 6$ months before switching to faster aspart at study start (week 0; baseline). Primary endpoint was $\mathrm{HbA}_{1 \mathrm{c}}$ change from baseline to week 24 . Exploratory endpoint was change in iscCGM metrics from baseline to week 24.

Results: Overall, 243 patients were included (55.6\% male), with mean age/diabetes duration, 49.9/18.8 years; mean $\mathrm{HbA}_{1 \mathrm{c}}, 8.1 \%$. By week $24, \mathrm{HbA}_{1 \mathrm{c}}$ had decreased by $0.19 \%(-2.1 \mathrm{mmol} / \mathrm{mol}, P<0.0001)$ with no mean change in insulin doses or basal/bolus insulin ratios. For patients with sufficient available iscCGM data $(n=92)$ : "time in range" (TIR; 3.9-10.0 mmol/L) increased from $46.9 \%$ to $50.1 \%(P=0.01)$, corresponding to an increase of $46.1 \mathrm{~min} /$ day; time in hyperglycemia decreased from $49.1 \%$ to $46.1 \%(>10.0 \mathrm{mmol} / \mathrm{L}, P=0.026)$ and $20.4 \%$ to $17.9 \%(>13.9 \mathrm{mmol} / \mathrm{L}, P=0.013)$, corresponding to $43.5(P=0.024)$ and $35.6(P=0.015)$ fewer minutes per day on average spent in these ranges, respectively; no change for time in hypoglycemia $(<3.9$ and $<3.0 \mathrm{mmol} / \mathrm{L})$. Mean interstitial and postprandial glucose improved from 10.4 to $10.1 \mathrm{mmol} / \mathrm{L}(P=0.035)$ and 11.9 to $11.0 \mathrm{mmol} / \mathrm{L}(P=0.002)$, respectively.
\end{abstract}

Conclusion: Real-world switching to faster aspart in adults with T1D on MDI improved $\mathrm{HbA}_{1 \mathrm{c}}$, increased TIR, and decreased time in hyperglycemia without affecting time in hypoglycemia.

The GoBolus study: NCT03450863.

Keywords: Diabetes mellitus, Continuous glucose monitoring, Prandial insulin, Time-in-range.

\footnotetext{
${ }^{1}$ Diabetes Center for Children and Adolescents, Children's Hospital on the Bult, Hanover Medical School, Hanover, Germany.

${ }^{2}$ Novo Nordisk Pharma GmbH, Mainz, Germany.

${ }^{3}$ Schwerpunktpraxis für Diabetes und Ernährungsmedizin, Muenster, Germany.

${ }^{4}$ Medizinisches Versorgungszentrum im Altstadt-Carree Fulda GmbH, Fulda, Germany.

${ }^{5}$ Diabetes Clinic for Children and Adolescents, Muenster, Germany.

Parts of this study were presented as a poster presentation at the American Diabetes Association, 80th Scientific Sessions (Virtual Meeting) on June 12-16, 2020, Chicago, IL, USA.

(C) Thomas Danne, et al., 2020; Published by Mary Ann Liebert, Inc. This Open Access article is distributed under the terms of the Creative Commons License (http://creativecommons.org/licenses/by/4.0), which permits unrestricted use, distribution, and reproduction in any medium, provided the original work is properly credited.
} 


\section{Introduction}

$\mathbf{T}$ YPE 1 DIABETES (T1D) IS a chronic disease, characterized by absent or near absent beta-cell function, that requires insulin therapy. ${ }^{1}$ Patients with T1D are often treated with a regimen of multiple daily injections (MDI), comprising a long-acting basal component and a mealtime bolus component of short-acting insulin. ${ }^{1}$ The development of fasteracting insulin analogs for prandial dosing increases the treatment options for patients, potentially offering improved glycemic control and more flexibility with dose timing. ${ }^{1-5}$

Fast acting insulin aspart (faster aspart) is an enhanced formulation of insulin aspart (IAsp) containing the excipients niacinamide and L-arginine. ${ }^{6}$ Niacinamide increases the initial abundance of IAsp monomers after subcutaneous administration and mediates a transient, local vasodilatory effect. $^{6}$ A post hoc analysis of pooled data from six pharmacology trials in adults with T1D showed that faster aspart had an $\sim 5$-min earlier onset of appearance in the circulation, a twofold greater early insulin exposure, and a $74 \%$ greater early glucose-lowering effect in the first $30 \mathrm{~min}$ after injection, compared with IAsp. ${ }^{7}$ The efficacy and safety of faster aspart was investigated in the "onset" clinical trial program and, as part of this, its clinical utility in adult patients receiving MDI was established. ${ }^{2,4,8-10}$ Faster aspart was demonstrated to be noninferior to IAsp with respect to reductions in $\mathrm{HbA}_{1 \mathrm{c}}{ }^{2,8,11}$ Adult and pediatric patients with T1D and adults with type 2 diabetes who were treated with faster aspart experienced improved postprandial glucose (PPG) control and similar or lower rates of hypoglycemia compared with IAsp. ${ }^{2,8,11}$ Moreover, the magnitude of improvements in PPG control with faster aspart versus IAsp are similar to those observed when earlier trials compared short-acting insulin analogs with regular human insulin. ${ }^{12,13}$ Faster aspart therefore represents an important addition to the available treatment options for T1D. Other ultrafast acting insulins are also in development. ${ }^{14}$

The "onset" clinical trials used a combination of selfmeasured blood glucose monitoring and continuous glucose monitoring (CGM) to investigate the efficacy of faster aspart, ${ }^{2,3,5,8-11}$ with the latter, newer method being particularly useful to examine PPG control. CGM yields real-time information that allows identification of acute glycemic excursions, and analysis of intra- and interday glucose variability and time in range (TIR). ${ }^{15}$ Hence, these data are a valuable addition to traditional $\mathrm{HbA}_{1 \mathrm{c}}$ measurements. "Intermittent-scanning continuous glucose monitoring", (iscCGM) systems (also known as flash glucose monitoring) are becoming widely used as a self-management tool for patients, since they are easy to use and do not require fingerprick calibration. ${ }^{16,17}$ All together, these systems provide comprehensive, meaningful data that allow patients and healthcare professionals to react faster to glucose perturbations or trends and better enable them to closely monitor the outcomes of treatment adjustments or switches.

Most of the clinical data on faster aspart are from the controlled "onset" treat-to-target clinical trials; there is limited real-world evidence of the impact of faster aspart. The GoBolus study, which is the first real-world evidence study investigating faster aspart, was therefore designed to analyze the real-world effectiveness and safety of faster aspart in patients with T1D on MDI who were using iscCGM, and thus examine if the observations of the "onset" trials translate to clinical practice. An exploratory analysis also investigated the change in iscCGM metrics from baseline.

\section{Materials and Methods}

\section{Study design}

GoBolus (NCT03450863) was a 24-week, multicenter, single-arm, observational study conducted in Germany with retrospective data extraction and prospective data collection to assess the effectiveness of faster aspart in adult patients with T1D using iscCGM. The study was noninterventional, as prescription of faster aspart was independent of this study and at the discretion of the treating physician as part of their usual clinical practice. Diagnostic or monitoring procedures outside of usual clinical practice were not applied.

The study included three visits in line with the local clinical practice: an initiation visit, which was the starting point of treatment with faster aspart (visit 1; week 0; baseline), a mid-study visit (visit $2 ; 12 \pm 4$ weeks), and an end-of-study visit (visit 3; $24 \pm 4$ weeks). The study recruited patients who were to be started on faster aspart as part of their usual clinical practice, and it was initiated on March 22, 2018 (first patient, first visit) and completed on September 4, 2019 (last patient, last visit).

The study was conducted in accordance with International Conference on Harmonization Good Clinical Practice Guidelines $^{18}$ and the Declaration of Helsinki. ${ }^{19}$ Informed consent was obtained before any trial-related activities. Patients received complete information about the study both verbally and in writing. The study received a professional legal consultation according to $\S 15$ of the professional regulations for doctors. The primary consultation was done by the Ethics Committee of the state medical association of Bavaria with the registration number 17089 on January 25, 2018. Further respective approvals were received by other relevant regional Ethics Committees.

\section{Participants}

The inclusion and exclusion criteria for study participation were sufficiently broad to help generalizability of study results to the wider adult population of patients with T1D using faster aspart treatment in local healthcare settings in Germany.

Patients included were adults ( $\geq 18$ years old), diagnosed with T1D at least 1 year before study inclusion and on stable insulin treatment with MDI for at least the previous 6 months before inclusion in this study, with $\mathrm{HbA}_{1 \mathrm{c}}$ between $7.5 \%$ and $9.5 \%$ at the latest reading (in the last 3 months). The upper limit of $\mathrm{HbA}_{1 \mathrm{c}}$ levels was set to $9.5 \%$ to exclude patients who needed basal insulin optimization, as the study aimed to observe the impact of the change in bolus insulin treatment. Patients were also required to be regular users (defined as usage on a monthly basis) of iscCGM for at least 6 months before study inclusion (visit 1; week 0; baseline).

Key exclusion criteria included antidiabetic treatment intensification (defined as adding new antidiabetic medication to previous treatment regimen) during the 3 months before study start, and women who were pregnant, breast feeding, or where pregnancy during the study was a possibility. 
The analysis sets were defined as follows: the full analysis set (FAS) included all enrolled patients, defined as all those who had signed an informed consent for the study, excluding screening failures; the safety analysis set (SAS) included all patients who had received at least one dose of study treatment; the effectiveness analysis set (EAS) included all patients from the SAS without relevant protocol deviations and who continued on treatment until visit 3 of the study; and for the primary endpoint EAS (EAS-P), the visit window for visit 3 was extended in a stepwise manner on a weekly basis in line with the statistical analysis plan, until the required group size for the primary endpoint was reached after $24 \pm 7$ weeks. Protocol deviations were as follows: the latest $\mathrm{HbA}_{1 \mathrm{c}}$ value was not measured in the 3 months before study inclusion (week 0 ; visit 1; baseline); or $\mathrm{HbA}_{1 \mathrm{c}}$ assessment was after treatment initiation with faster aspart; or $\mathrm{HbA}_{1 \mathrm{c}}$ value at baseline was $<7.5 \%$ or $>9.5 \%$; or visit 3 not performed at $24 \pm 4$ weeks.

\section{Procedures}

Patients were treated with commercially available faster aspart as bolus insulin injections (MDI). Dosing with faster aspart was individual and determined by the treating physician in accordance with the needs of the patient. All other antidiabetic medications were prescribed at the discretion of the treating physician under routine clinical practice conditions.

The iscCGM monitoring device used in this study was the Freestyle Libre ${ }^{\circledR}$ (Abbott, IL, USA). A period of at least 14 days of retrospective data collection (a full 2 weeks sensor download as csv file with at least $80 \%$ completeness of the data) was required to allow sufficient analysis of isCGM data before each visit. If a data set was found with less than $80 \%$ completeness, the previous date of sensor change before the evaluated period was chosen to determine the start of the previous measurement period. To find a period with sufficient completeness, this process was performed up to three times at baseline (visit 1; week 0), then up to two times at weeks 12 (visit 2) and 24 (visit 3). The iscCGM data were checked for the following: to identify systematic gaps (data missing for more than $4.8 \mathrm{~h}$ on the same period of 3 or more days) and possible wrong measurements (e.g., values of $>22.2 \mathrm{mmol} / \mathrm{L}$ [ $>400 \mathrm{mg} / \mathrm{dL}$ ] were checked); to verify that times and dates were in the expected format and aligned with visit dates/sensor changes; to verify that iscCGM units were the same for all participants; and to check for extra data and duplicate time stamps.

\section{Outcome measures}

The primary endpoint of this study was change in $\mathrm{HbA}_{1 \mathrm{c}}$ from baseline (week 0) to week 24. In cases where fasting plasma glucose $(\mathrm{FPG}) / \mathrm{HbA}_{1 \mathrm{c}}$ was not measured at the initiation visit (visit 1; week 0 ; baseline), the latest $\mathrm{HbA}_{1 \mathrm{c}}$ and FPG measurement in the health record within the previous 3 months were used as baseline measurements. $\mathrm{HbA}_{1 \mathrm{c}}$ was measured locally at each site's laboratory.

Secondary endpoints included change from baseline (week 0) to week 12 in $\mathrm{HbA}_{1 \mathrm{c}}$; change from baseline to week 12 and to week 24 in laboratory-measured FPG; and change from baseline to week 24 in the following: total daily basal insulin, bolus insulin, and basal/bolus ratio (defined as total daily basal insulin [U] divided by total daily bolus insulin), timing of insulin administration in relationship to the start of the meal (measured in minutes), total treatment satisfaction score in the Diabetes Treatment Satisfaction Questionnaire (DTSQ) and total treatment-related impact in the Treatment Related Impact Measure for Diabetes (TRIM-D) questionnaire.

Exploratory endpoints included reasons for initiating faster aspart, reasons for premature discontinuation of faster aspart, and the change in iscCGM data from baseline (week 0 ) to week 12 and week 24 for the FAS dataset. Analyses of iscCGM data with the EAS dataset were done as post hoc analyses.

iscCGM data were described by means of consolidated ambulatory glucose profile (as recommended by international consensus $^{20}$ ) and endpoints included mean interstitial glucose (measured within $24 \mathrm{~h}$, day and night), TIR (referred to as the time spent in the target glucose range of the patient: $3.9-10 \mathrm{mmol} / \mathrm{L}[70-180 \mathrm{mg} / \mathrm{dL}])$, time spent in hypoglycemia $(<3 \mathrm{mmol} / \mathrm{L}[<54 \mathrm{mg} / \mathrm{dL}],<3.9 \mathrm{mmol} / \mathrm{L}[<70 \mathrm{mg} / \mathrm{dL}])$, time spent in hyperglycemia $(>10 \mathrm{mmol} / \mathrm{L}[>180 \mathrm{mg} / \mathrm{dL}]$, $>13.9 \mathrm{mmol} / \mathrm{L}[>250 \mathrm{mg} / \mathrm{dL}]$ ), $\mathrm{PPG}$ (defined as the average glucose raise of two consecutive measurements of a value $2 \mathrm{mmol} / \mathrm{L}[36 \mathrm{mg} / \mathrm{dL}$ ] higher than fasting preprandial glucose [FPPG] occurring within $3 \mathrm{~h}$ of FPPG), FPPG (defined as the first glucose value after night time [after 05:59 am] before first glucose raise occurring before 09:00 am), estimated $\mathrm{HbA}_{1 \mathrm{c}}$, number and duration of hypoglycemic episodes (glucose alert value $<3.9 \mathrm{mmol} / \mathrm{L}[<70 \mathrm{mg} / \mathrm{dL}$ ], and clinically significant hypoglycemia $<3 \mathrm{mmol} / \mathrm{L}[<54 \mathrm{mg} / \mathrm{dL}]$ ) in $24 \mathrm{~h}$. TIR and time in hypo- and hyperglycemia are expressed as the percentage of measurements that are in each of the given glucose ranges and the average minutes per day spent in the given ranges. ${ }^{15}$ Hypoglycemia was defined as at least two consecutive readings at 15 -min intervals, $<3 \mathrm{mmol} / \mathrm{L}$ $(54 \mathrm{mg} / \mathrm{dL})$, with the end of an episode represented by two readings at or higher than this threshold. Glycemic variability was evaluated by analyzing the coefficient of variation of the mean daily glucose and mean amplitude of glucose excursion (MAGE).

At each visit, hospitalizations for diabetic ketoacidosis or severe hypoglycemia, the number of nonserious hypoglycemic episodes, serious adverse reactions (SAR), and fatal events or pregnancies were recorded.

Measurements taken at visit 1 (or within previous 3 months of visit 1 for $\mathrm{HbA}_{1 \mathrm{c}}$ and FPG) are hereafter referred to as baseline measurements.

\section{Statistical analysis}

The primary and secondary endpoints were analyzed using a paired $t$-test to assess the statistical significance of the mean change in each parameter from baseline to week 12 or 24 .

TRIM-D ${ }^{21,22}$ includes 28 items that are grouped into 5 domains (treatment burden, daily life, diabetes management, compliance, and psychological health), where items are scored from 1 to 5 (higher scores indicate a better outcome). Total treatment-related impact was computed by adding all items and a transformation such that values ranged from 0 to 100. Total treatment-related impact could only be computed if all of the domains could be scored. DTSQ $^{23,24}$ was a six-item patient-reported outcome (based on treatment satisfaction and perceived frequency of hyperand hypoglycemia) scored on a scale from 0 to 6 (higher scores indicate a better outcome). If one or two items were 
not answered, total treatment satisfaction could still be computed. Comparisons in change from baseline in scores were performed with paired $t$-tests similar to that used for the primary analysis.

The iscCGM data consisted of the aggregated analysis of data from the patients. CGM metrics were calculated according to the international consensus on CGM metrics. ${ }^{20}$ All comparisons between visits were done by means of paired $t$-tests. All tests performed were two sided with a $5 \%$ level of significance.

Safety data, reasons for treatment initiation/premature discontinuation, and change in timing of insulin administration were summarized descriptively.

The study planned to enroll 220 patients with MDI, with assumption rates of $10 \%$ for screening failure and $15 \%$ for withdrawal (loss of a patient for any reason before completing the 24 weeks observation period).

\section{Results}

The study was conducted in 41 sites in Germany between March 2018 and September 2019. In total, 244 patients were enrolled and 241 were treated with faster aspart (Supplementary Fig. S1). The three main reasons given for initiating faster aspart were to improve the patient's blood glucose profile, insufficient $\mathrm{HbA}_{1 \mathrm{c}}$ adjustment on patient's current regimen, and improved time flexibility in bolus administration (Supplementary Table S1). The main reasons for premature discontinuation are shown in Supplementary Table S2 and include loss to follow-up, which accounted for $4.1 \%$ of patients from the SAS.
Baseline characteristics are summarized in Table 1. Of the 155 patients who comprised the EAS, iscCGM data with sufficient data completeness were available for 92 patients (Supplementary Fig. S1; Table 1). Data presented for effectiveness endpoints are from the EAS (EAS-P for primary endpoint, iscCGM-EAS for exploratory endpoints), while safety data are from the SAS $(n=241)$. Data concerning effectiveness endpoints for the FAS can be found in the Supplementary Materials (Supplementary Table S3 and Supplementary Figs. S2-S4).

\section{Primary effectiveness endpoint: change in $\mathrm{HbA}_{1 \mathrm{c}}$ over time}

Patients receiving faster aspart experienced a significant mean decrease of $-0.19 \%(95 \% \mathrm{CI}:-0.27$ to -0.10 ; $P<0.0001)(-2.1 \mathrm{mmol} / \mathrm{mol}(95 \% \mathrm{CI}:-3.0$ to -1.1$)$ in their $\mathrm{HbA}_{1 \mathrm{c}}$ from $8.1 \%(64.8 \mathrm{mmol} / \mathrm{mol})$ at baseline to $7.9 \%$ $(62.8 \mathrm{mmol} / \mathrm{mol})$ at week 24 (EAS-P; Fig. 1). The $\mathrm{HbA}_{1 \mathrm{c}}$ at week 12 was also $7.9 \%(62.8 \mathrm{mmol} / \mathrm{mol})$, which was also a significant reduction from baseline $(-0.15 ; 95 \% \mathrm{CI}$ : -0.24 to $-0.07 ; P=0.001)(-1.6 \mathrm{mmol} / \mathrm{mol}(95 \% \mathrm{CI}:-2.6$ to -0.8$)$.

\section{Intermittent-scanning continuous glucose monitoring data}

There were significant reductions from baseline to week 24 in mean PPG $(-0.8 \mathrm{mmol} / \mathrm{L}[-15.1 \mathrm{mg} / \mathrm{dL}(\mathrm{SD} 43.0)]$, $P=0.002)$, FPPG $(-0.8 \mathrm{mmol} / \mathrm{L}[-13.8 \mathrm{mg} / \mathrm{dL}(\mathrm{SD} 44.0)]$, $P=0.005)$, and estimated $\mathrm{HbA}_{1 \mathrm{c}}(-0.2 \%$ [0.8], 2.2 [8.7] $\mathrm{mmol} / \mathrm{mol}, P=0.035$ ) (Table 2, FAS data in Supplementary

Table 1. Baseline Characteristics of Patients, Full Analysis Set, and Effectiveness Analysis Set

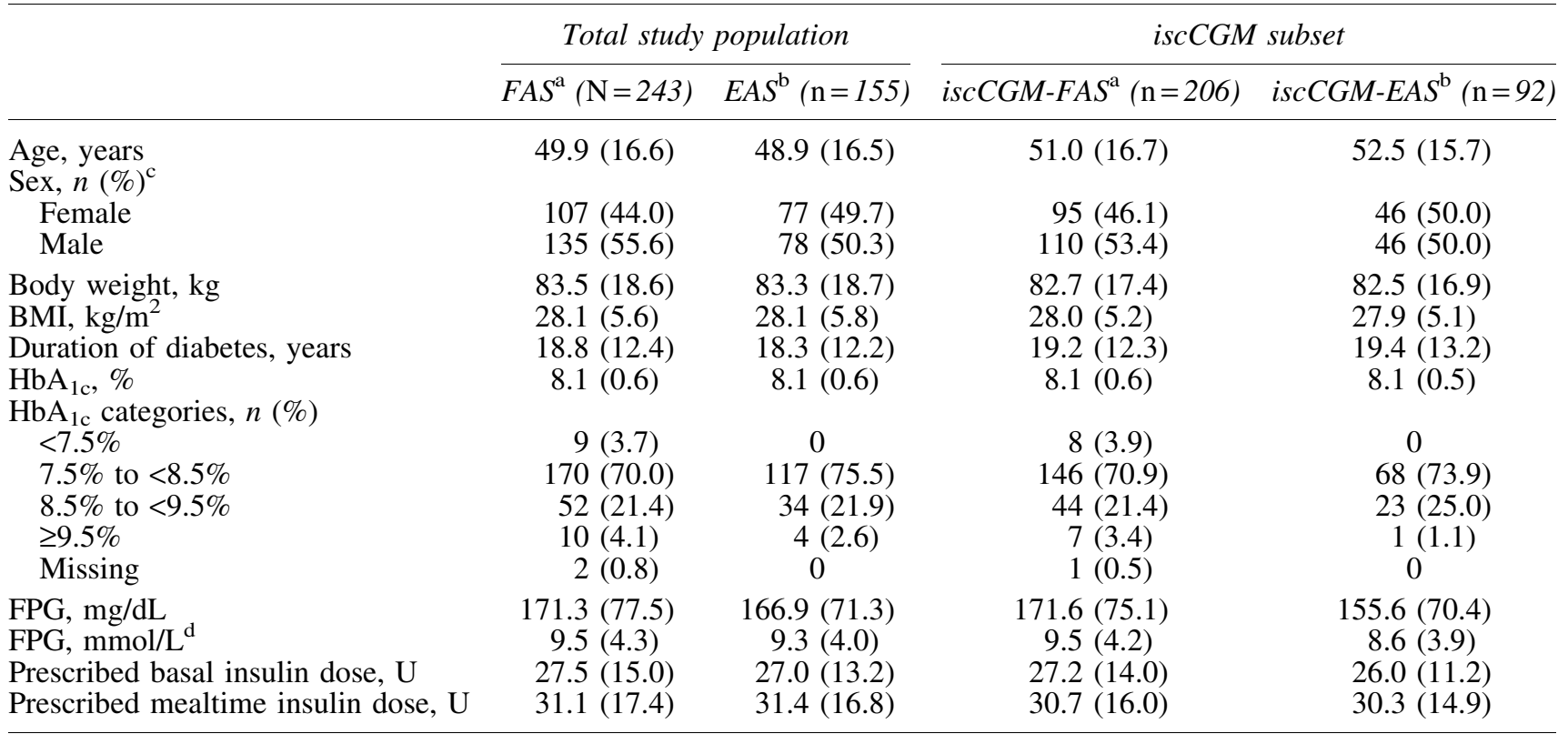

Data are mean $( \pm \mathrm{SD})$ unless otherwise stated.

${ }^{a}$ FAS: included all enrolled patients, defined as all those who had signed an informed consent for the study, excluding screening failures.

${ }^{b}$ EAS: included all patients from the SAS without relevant protocol deviations and who continued on treatment until visit 3 of the study.

${ }^{c}$ One patient had this data missing from the FAS of the iscCGM set.

${ }^{\mathrm{d}}$ Calculated by dividing $\mathrm{mg} / \mathrm{dL}$ data by 18.02 .

BMI, body mass index; EAS, effectiveness analysis set; FAS, full analysis set; FPG, fasting plasma glucose; HbA $1 \mathrm{c}$, glycated hemoglobin; iscCGM, intermittent-scanning continuous glucose monitoring; iscCGM-EAS, patients with intermittent-scanning continuous glucose monitoring data in the effectiveness analysis set; iscCGM-FAS/EAS, patients with intermittent-scanning continuous glucose monitoring data in the full/effectiveness analysis set; $n$, number of patients; SAS, safety analysis set; SD, standard deviation; $\mathrm{U}$, international unit. 


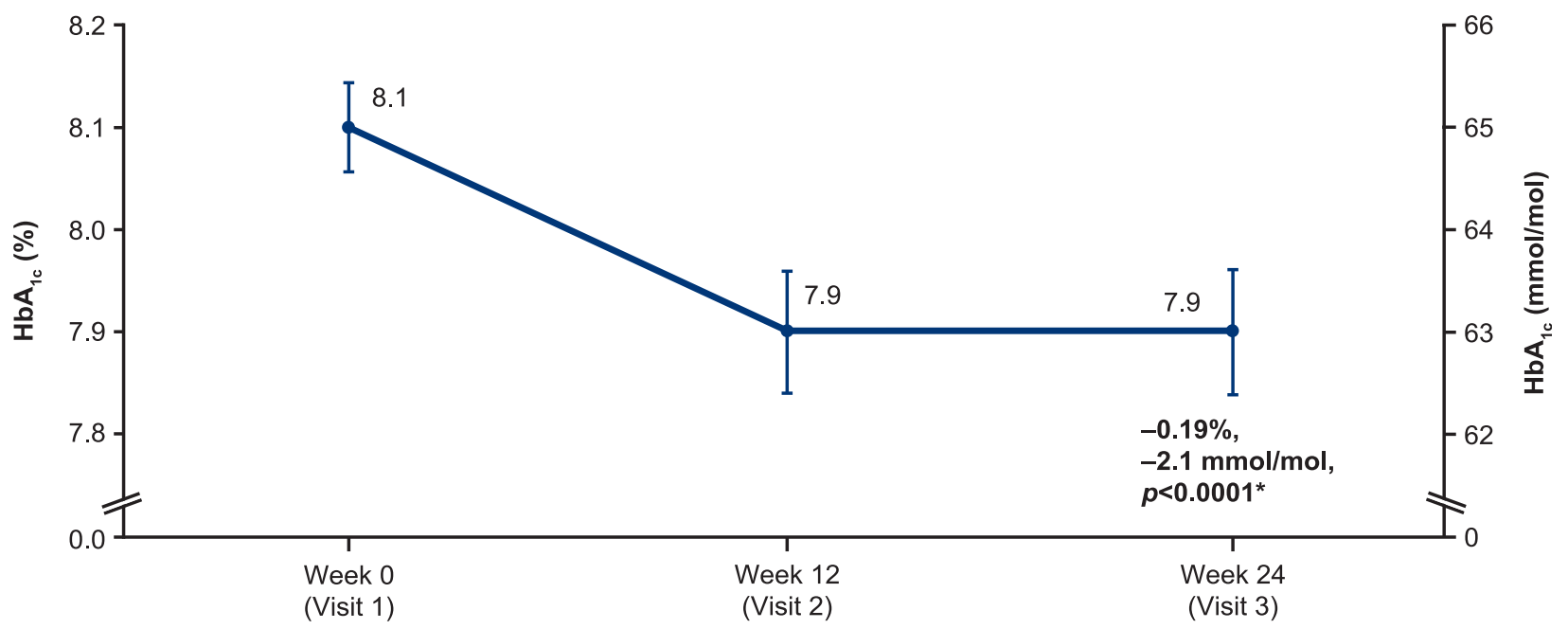

FIG. 1. Mean $\mathrm{HbA}_{1 \mathrm{c}}$, EAS-P $(n=170)$. *Mean of the pairwise differences between visits per patient; values depicted are mean \pm standard error. EAS-P, extended effectiveness analysis set for the primary endpoint; HbA $1 \mathrm{c}$, glycated hemoglobin.

Table 2. Aggregated Intermittent-Scanning Continuous Glucose Monitoring Results Over Time, Effectiveness Analysis Set

\begin{tabular}{|c|c|}
\hline & $i s c C G M-E A S^{\mathrm{a}}$ \\
\hline \multicolumn{2}{|l|}{ Mean (SD) postprandial glucose, $\mathrm{mmol} / \mathrm{L}[\mathrm{mg} / \mathrm{dL}]$} \\
\hline Baseline; week 0 & $11.9(2.3)[213.7(41.7)]$ \\
\hline Week 12 & $11.3(2.3)[204.5(40.8)]$ \\
\hline Week 24 & $11.0(2.4)[198.3(43.9)]$ \\
\hline Change in postprandial glucose from week 0 to week 24 & $-0.8(2.4)[-15.1(43.0)], P=0.002$ \\
\hline \multicolumn{2}{|l|}{ Mean (SD) fasting preprandial glucose (before $9 \mathrm{am}$ ), $\mathrm{mmol} / \mathrm{L}[\mathrm{mg} / \mathrm{dL}]$} \\
\hline$N^{\mathrm{b}}$ & $89^{\mathrm{b}}$ \\
\hline Baseline; week 0 & $8.6(2.4)[155.3(43.1)]$ \\
\hline Week 12 & $8.1(2.3)[146.2(41.5)]$ \\
\hline Week 24 & $7.8(2.4)[141.0(43.0)]$ \\
\hline Change in fasting preprandial glucose from week 0 to week 24 & $-0.8(2.4)[-13.8(44.0)], P=0.005$ \\
\hline \multicolumn{2}{|l|}{ Mean (SD) estimated $\mathrm{HbA}_{1 \mathrm{c}}, \%$} \\
\hline & 92 \\
\hline Baseline; week 0 & $8.2(1.0)$ \\
\hline Week 12 & $8.1(1.1)$ \\
\hline Week 24 & $8.0(1.0)$ \\
\hline Change in estimated $\mathrm{HbA}_{1 \mathrm{c}}$ from week 0 to week 24 & $-0.2(0.8), P=0.035$ \\
\hline \multicolumn{2}{|l|}{ Mean (SD) interstitial glucose, $\mathrm{mmol} / \mathrm{L}[\mathrm{mg} / \mathrm{dL}]$} \\
\hline$N$ & 92 \\
\hline Baseline; week 0 & $10.4(1.6)[187.6(28.1)]$ \\
\hline Week 12 & $10.3(1.8)[185.3(32.2)]$ \\
\hline Week 24 & $10.1(1.5)[182.2(27.9)]$ \\
\hline Change in mean interstitial glucose from week 0 to week 24 & $-0.3(1.3)[-5.4(24.2)], P=0.035$ \\
\hline \multicolumn{2}{|l|}{ Mean (SD) coefficient of variation, \% } \\
\hline$N$ & 92 \\
\hline Baseline; week 0 & $38.7(6.6)$ \\
\hline Week 12 & $37.9(5.8)$ \\
\hline Week 24 & $38.4(5.6)$ \\
\hline Change in mean coefficient of variation from week 0 to week 24 & $-0.4(5.7), P=0.541$ \\
\hline \multicolumn{2}{|l|}{ Mean (SD) MAGE } \\
\hline$N$ & 92 \\
\hline Baseline; week 0 & $162.0(33.9)$ \\
\hline Week 12 & $155.2(34.3)$ \\
\hline Week 24 & $154.4(29.4)$ \\
\hline Change in MAGE from week 0 to week 24 & $-7.5(32.8), P=0.03$ \\
\hline
\end{tabular}

${ }^{a}$ EAS: included all patients from the SAS without relevant protocol deviations and who continued on treatment until visit 3 of the study. ${ }^{\mathrm{b}} N$ numbers for PPG and FPPG were 89 (baseline), 88 (weeks 12 and 24), and 86 (change in value). $\mathrm{mmol} / \mathrm{L}$ data calculated by dividing $\mathrm{mg} / \mathrm{dL}$ data by 18.02 . MAGE, mean amplitude of glycemic excursions. 


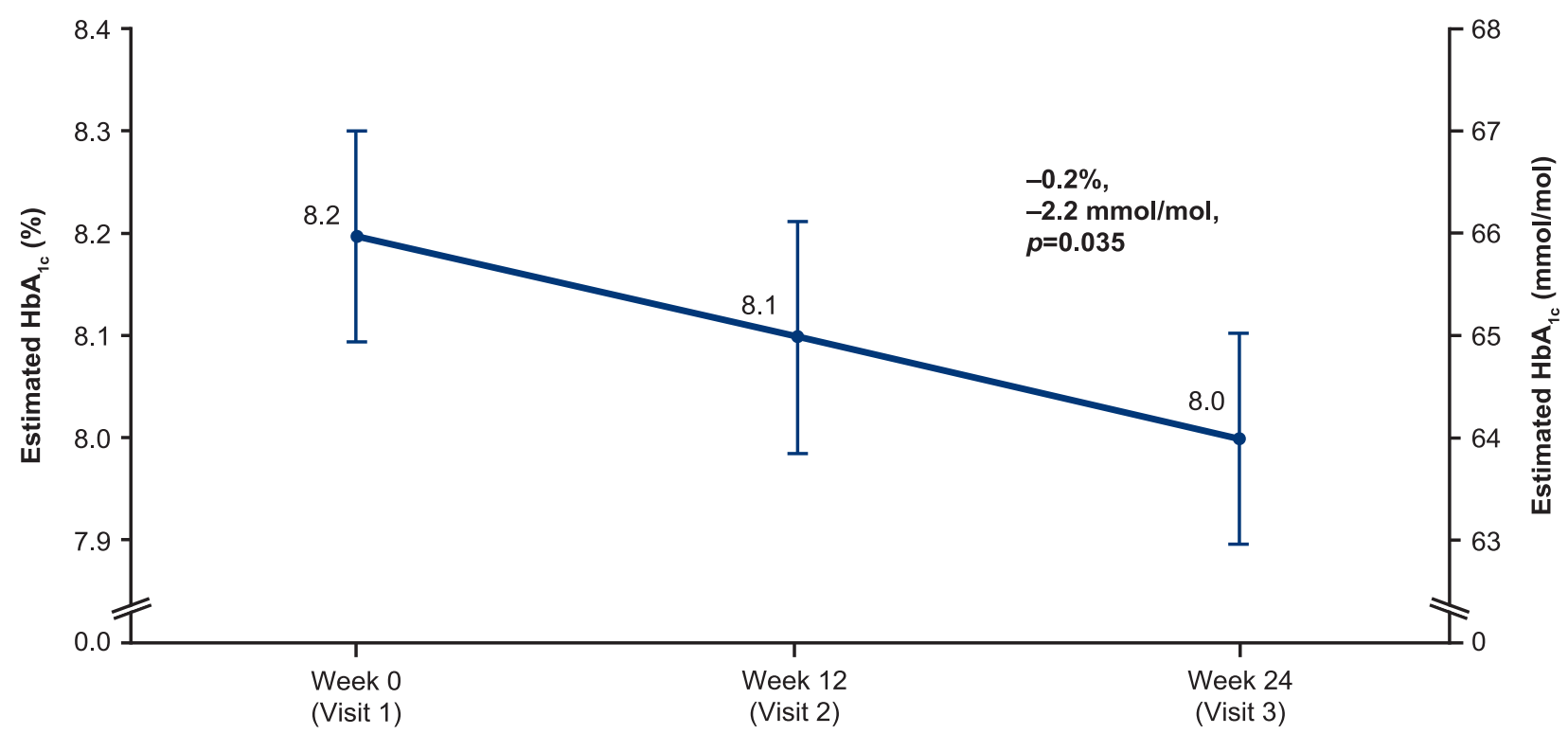

FIG. 2. Mean estimated $\mathrm{HbA}_{1 \mathrm{c}}$, EAS-iscCGM $(n=92)$. EAS-iscCGM, effectiveness analysis set patients with sufficient intermittent-scanning continuous glucose monitoring data available; $\mathrm{HbA}_{1 \mathrm{c}}$, glycated hemoglobin.

Table S3). Estimated $\mathrm{HbA}_{1 \mathrm{c}}$ is shown in Figure 2 (EAS) and Supplementary Figure S2 (FAS).

The percentage of measurements within each glucose range is shown in Figure 3 (FAS data in Supplementary Fig. S3). The changes between baseline to week 24 were as follows: the percentage of measurements in the target glucose range $(3.9-10 \mathrm{mmol} / \mathrm{L}[70-180 \mathrm{mg} / \mathrm{dL}])$ increased from $46.9 \%$ to $50.1 \%(P=0.01)$, the percentage of measurements that were $>10 \mathrm{mmol} / \mathrm{L}(>180 \mathrm{mg} / \mathrm{dL})$ decreased from $49.1 \%$ to $46.1 \%(P=0.026)$, while those that were $>13.9 \mathrm{mmol} / \mathrm{L}$ $(>250 \mathrm{mg} / \mathrm{dL})$ decreased from $20.4 \%$ to $17.9 \%(P=0.013)$ and the percentage of measurements in low or very low glucose ranges remained virtually unchanged. The change in average minutes per day spent in each assessed glucose range is shown in Figure 4 (FAS data in Supplementary Fig. S4). The average minutes per day spent in target range significantly increased by $46.1 \mathrm{~min}(P=0.009)$ and was accompanied by a significant decrease in time spent in hyperglycemia ( $-43.5 \mathrm{~min}, P=0.024$ for $>10 \mathrm{mmol} / \mathrm{L}[>180 \mathrm{mg} / \mathrm{dL}]$ threshold and $-35.6 \mathrm{~min}, P=0.015$ for $>13.9 \mathrm{mmol} / \mathrm{L}[>250 \mathrm{mg} / \mathrm{dL}]$ threshold), while time spent in hypoglycemia remained virtually unchanged compared with baseline.

There were no significant differences in the mean overall number of hypoglycemic episodes, including very low
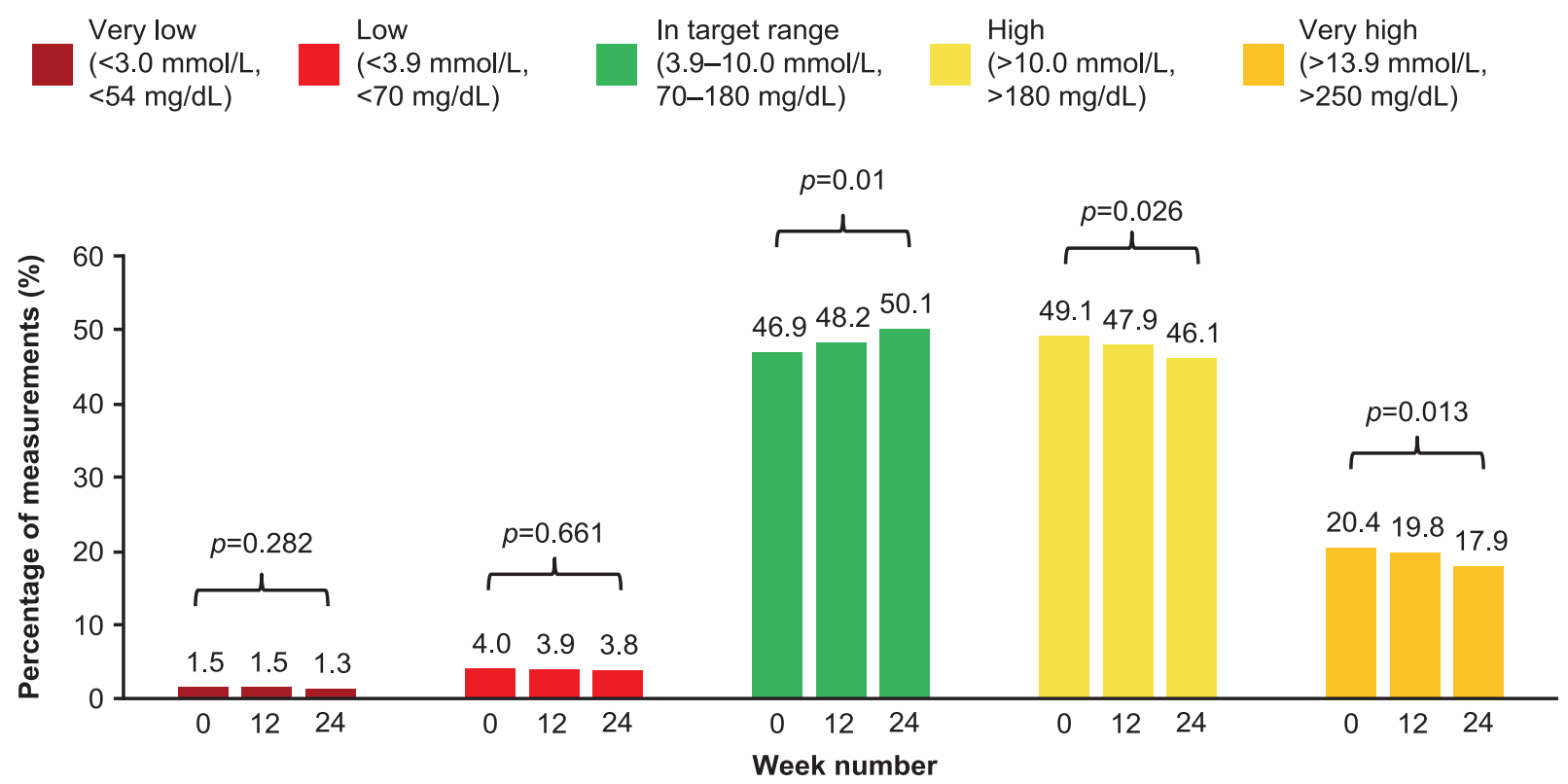

FIG. 3. Average percentage of measurements within each glucose range, iscCGM-EAS ( $n=92)$. iscCGM-EAS, effectiveness analysis set patients with sufficient intermittent-scanning continuous glucose monitoring data available. 


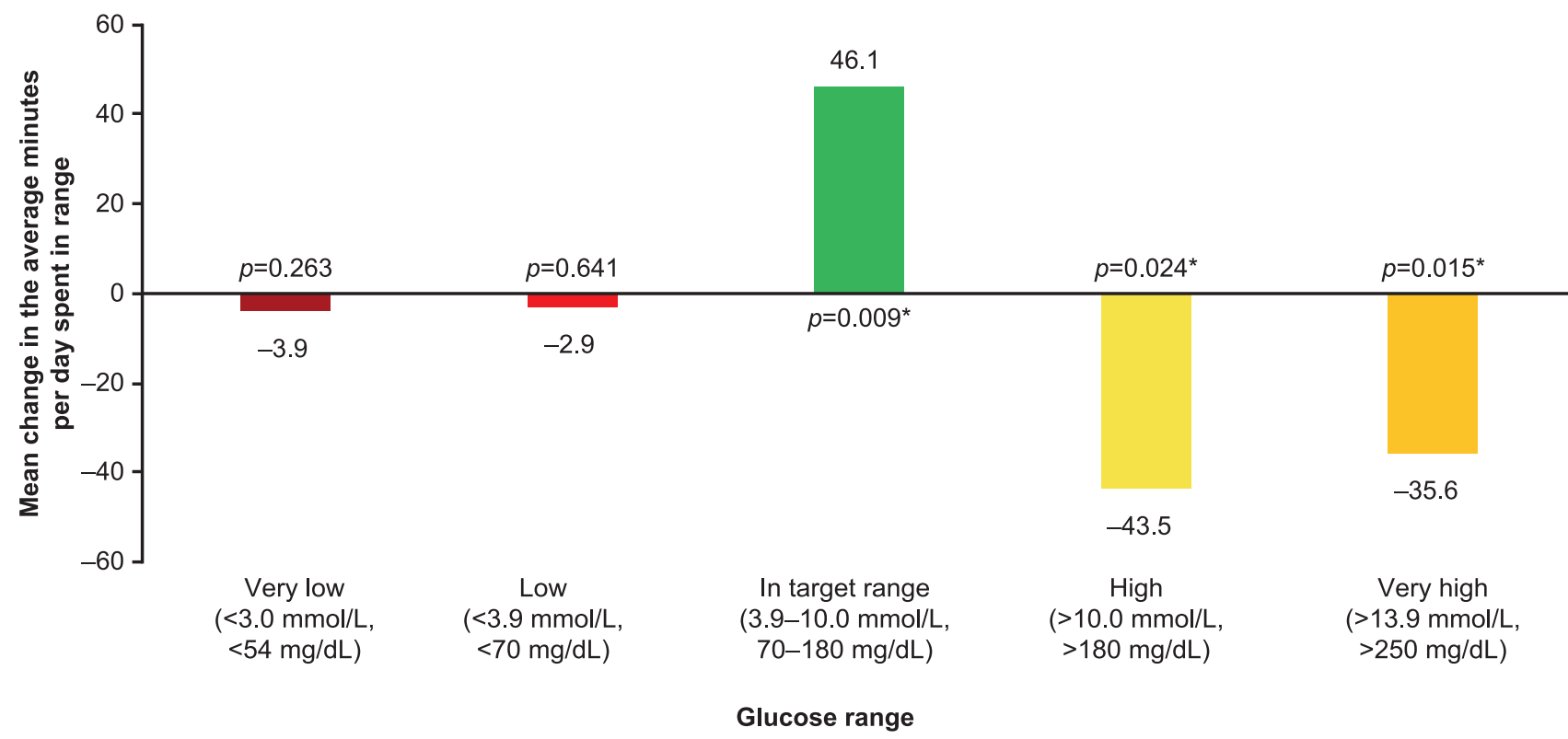

FIG. 4. Mean change from baseline to week 24 in average minutes per day spent in each glucose range, iscCGM-EAS $(n=92) . *$ Significant difference versus baseline. EAS, effectiveness analysis set.

glucose episodes $(<3.0 \mathrm{mmol} / \mathrm{L}[<54 \mathrm{mg} / \mathrm{dL}])$ and low glucose episodes $(<3.9 \mathrm{mmol} / \mathrm{L}[<70 \mathrm{mg} / \mathrm{dL}])$. Overall, the mean interstitial glucose was reduced from baseline at weeks 12 and 24 (Table 2). This reduction was most pronounced at week $24(-0.3 \mathrm{mmol} / \mathrm{L}$ [ $-5.4 \mathrm{mg} / \mathrm{dL} ; \mathrm{SD} 24.2], P=0.035$, EAS [Table 2, FAS data in Supplementary Table S3]).

There was only a small mean change in coefficient of variation at weeks 12 and 24 compared with baseline $(-0.8$ at week $12,-0.4$ at week 24; not significant; Table 2). MAGE was significantly reduced at weeks 12 and $24(-6.7$ at week $12[P=0.03],-7.5$ at week $24[P=0.03]$; Table 2$)$.

\section{Secondary endpoints}

Fasting plasma glucose. The change in laboratorymeasured FPG from baseline to week $24(-0.2 \mathrm{mmol} / \mathrm{L}$ [ $-3.63 \mathrm{mg} / \mathrm{dL} ; 95 \% \mathrm{CI}$ : -19.46 to 12.21$]$ ) and week 12 ( $-0.4 \mathrm{mmol} / \mathrm{L}[-8.10 \mathrm{mg} / \mathrm{dL} ; 95 \% \mathrm{CI}:-22.06$ to 5.85$])$ was small and not statistically significant.
Insulin dose. The change from previous therapy to week 24 in basal $(0.5 \mathrm{U}$ [95\% CI: -0.42 to 1.41$])$ and bolus $(-1.2 \mathrm{U}$ [95\% CI: -3.00 to 0.71$]$ ) insulin dose was not statistically significant. Similarly, there was no significant difference in the basal/bolus insulin ratio from baseline to week $24(-8.9$ [95\% CI -40.36 to 22.65]).

After switching to faster aspart, a small number of patients changed the timing of bolus dosing to after the start of the meal: $3.2 \%(5 / 155)$ at baseline compared with $9.2 \%(14 / 153)$ at week 24 .

Patient reported outcomes. Quality of life improved from baseline to week 24: the total DTSQ score in the EAS increased by 1.7 (95\% CI: $0.71-2.72 ; P=0.001)$ and total TRIM-D score by 5.8 (95\% CI: $4.08-7.53 ; P<0.0001)$ (Table 3 for EAS and FAS).

Safety data. Overall, five patients reported six SARs until study completion: five SARs in four patients were

Table 3. Baseline, Week 24, and Change from Baseline to Week 24 Scores for the Diabetes Treatment Satisfaction Questionnaire and the Treatment Related Impact Measure for Diabetes Questionnaire, Full Analysis Set, and Effectiveness Analysis Set

\begin{tabular}{|c|c|c|c|c|c|}
\hline & Baseline & Week 24 & $\begin{array}{c}\text { Change from baseline } \\
\text { to week } 24\end{array}$ & $95 \% C I$ & $\mathrm{P}$ \\
\hline \multicolumn{6}{|l|}{ FAS $^{\mathrm{a}}$} \\
\hline Diabetes treatment satisfaction questionnaire & $27.8(5.6)$ & $29.4(5.5)$ & $1.7(6.2)$ & $0.71-2.72$ & 0.001 \\
\hline Treatment-related impact measure for diabetes & $69.6(11.1)$ & $75.4(11.7)$ & $5.8(9.7)$ & $4.08-7.53$ & $<0.0001$ \\
\hline \multicolumn{6}{|l|}{$\mathrm{EAS}^{\mathrm{b}}$} \\
\hline Diabetes treatment satisfaction questionnaire & $28.0(5.6)$ & $29.6(5.3)$ & $1.7(6.4)$ & $0.82-2.65$ & 0.001 \\
\hline Treatment-related impact measure for diabetes & $69.9(11.5)$ & $75.5(11.7)$ & $5.7(9.9)$ & $4.24-7.25$ & $<0.0001$ \\
\hline
\end{tabular}

Data are mean (SD) unless otherwise stated.

${ }^{a}$ FAS: included all enrolled patients, defined as all those who had signed an informed consent for the study, excluding screening failures.

${ }^{b}$ EAS: included all patients from the SAS without relevant protocol deviations and who continued on treatment until visit 3 of the study.

CI, confidence interval. 
nonfatal; one was fatal. The fatal event occurred after the patient (54-year-old male) went skiing and paragliding and subsequently experienced very low blood sugar. The cause of death was not listed and it is not possible to ascertain the patient's food intake or insulin dose that day. Of the nonfatal SARs, five (in four patients) were hospitalizations due to ketoacidosis (four events were moderate and one was severe in intensity; three events occurred alongside hyperglycemia of the same intensity as their diabetic ketoacidosis; all the events were assessed as unrelated to the study medication). Altogether, this does not change the current knowledge of the safety profile of faster aspart.

\section{Discussion}

This real-world study in Germany of adults with T1D confirmed findings from the regulatory "onset" trials of improved PPG control with faster aspart leading to statistically significant improvements in $\mathrm{HbA}_{1 \mathrm{c}}{ }^{2,3}$ and demonstrated improvements in TIR at 24 weeks without increasing time spent in hypoglycemia. The reduction in $\mathrm{HbA}_{1 \mathrm{c}}$ from baseline in this study is greater than the treatment difference observed between faster aspart and IAsp in the 26- and 52-week data from onset-1 and onset-8. ${ }^{3,4}$ Overall, FAS and EAS data correlated, indicating that these conclusions were not biased by selecting only the most adherent patients. Furthermore, the mean insulin doses and the mean basal/bolus ratio remained constant, supporting the findings from randomized controlled trials that patients could benefit solely from switching bolus insulin. ${ }^{3,4}$ Overall, our findings support the treatment switch to faster aspart in combination with iscCGM in insulin-experienced patients with T1D, particularly in those in need of better glycemic control and/or more flexible bolus administration.

In our study, a full 2-week sensor download with $80 \%$ complete data for each visit was available for 92 patients, and, therefore, an aggregated analysis of all single-patient iscCGM metrics could be performed. These metrics provided insights into the glycemic control of patients that would not be apparent from $\mathrm{HbA}_{1 \mathrm{c}}$ evaluations alone. ${ }^{20}$ The iscCGM data included significant reductions in mean interstitial glucose, PPG, and FPPG. Again, this is consistent with the improvements in PPG increment observed with faster aspart in clinical trials after a standardized liquid meal test in adults or in the CGM subgroup of children in onset 7 (both with MDI). ${ }^{3,11}$ The reduction in PPG likely accounts for much of the reduction in time in hyperglycemia, and hence the improved TIR. As the PPG control of patients with T1D is most critically influenced by their bolus insulin dose, these data provide evidence that faster aspart can help to improve overall glycemic control through reduced PPG excursions in a real-world setting.

While $\mathrm{HbA}_{1 \mathrm{c}}$ measurements are routinely included in clinical trials, alone, this metric fails to identify important changes in patients' blood glucose stability or frequent excursions in high or low glucose ranges. TIR is now recognized as an important parameter to measure in patients with diabetes, ${ }^{15,25-27}$ and several studies have demonstrated an association between TIR and the risk of diabetic complications. ${ }^{28-31}$ For example, Beck et al. demonstrated that the hazard rate for retinopathy progression increased by $64 \%$ for each $10 \%$ reduction in TIR (as calculated from seven-point self-measured blood glucose profiles). ${ }^{29}$ Therefore, the statistically significant improvements in TIR observed with initiation of faster aspart in this study are an important finding. Several parameters have been suggested as a measure of glycemic variability. ${ }^{20}$ While there was no significant difference in coefficient of variation, as an easy-to-calculate parameter, there was a statistically significant difference at week 24 in the MAGE, which is the classic marker of the amplitude of glucose fluctuations. This metric of glycemic variability uses a calculation that is "devoid of time component," that is to say that it focuses solely on the magnitude of the minimum to maximum glucose levels, regardless of the time it takes to transition from one extreme glucose level to the next. ${ }^{32}$ The impact of glycemic variability on long-term outcomes is controversial, however, some studies using CGM have demonstrated an association between glycemic variability and retinopathy, microalbuminuria, and neuropathy. ${ }^{33,34}$ This in turn highlights the value of reporting data from new technologies, such as iscCGM, to fully understand the effect of an intervention.

It is also important to report that real-world studies, such as GoBolus, look beyond $\mathrm{HbA}_{1 \mathrm{c}}$ and include patient-reported outcomes. ${ }^{35}$ The improved treatment satisfaction observed in the GoBolus DTSQ and TRIM-D analyses may reflect the totality of the improvement in glycemic control, but could also show a key advantage of faster aspart in that it offers increased dosing flexibility compared with other bolus insulins currently available. However, while being statistically significant, changes in DTSQ and TRIM-D were numerically small. As there is no predetermined minimally important change for many patient-reported outcome tools, including these questionnaires, the clinical meaning of these differences remains to be defined.

The key limitations of this study were due to the observational, open-label nature of the trial design, which could have affected study outcomes. Complete and reliable iscCGM data were only available for 92 participants in the EAS (63 had insufficient data quality); a limited sample but reflective of the observational design of our study. Real-world studies are more likely to be subject to bias such as selection bias, ${ }^{36}$ in this instance the decision to prescribe faster aspart may be due to patient or disease characteristics that would be controlled for in a randomized controlled trial. The nature of the study precluded a central laboratory; therefore differences, for example, in local $\mathrm{HbA}_{1 \mathrm{c}}$ measurement procedures cannot be ruled out; however, German national guidelines only allow quality-approved procedures, ${ }^{37,38}$ and therefore the data should be reliable. In addition, as the study did not have a control group, there is no way of confirming how clinical outcomes would be different if the patients had not switched to faster aspart. For example, we cannot exclude that some of the observed improvement in glycemic control was due to patients becoming more adept at using their iscCGM during the course of the study. However, this limitation has been addressed by including only patients who had regularly used their iscCGM device for at least 6 months before study start.

The key strength of the study was the enrolment of patients in a real-world setting, where the decision to initiate faster aspart was made independently of study participation. Inclusion criteria were wide enough to ensure that the study population resembles patients in German clinical practice and possibly countries with similar healthcare systems. 
Accordingly, the reported outcomes provide important insights into the impact of iscCGM and faster aspart on patients in the clinic, and they merit further study on a global level.

\section{Conclusion}

This study in Germany confirmed the clinical benefits of faster aspart reported in the "onset" randomized controlled trials, in a large population of patients with T1D receiving MDI, as well as highlighting the value of iscCGM for illustrating the impact of an intervention. Glycemic control was improved, as demonstrated by both $\mathrm{HbA}_{1 \mathrm{c}}$ and iscCGM data, by reducing glucose excursions and, overall, this was achieved without changing the type of basal insulin or the mean insulin dose.

\section{Acknowledgments}

The authors are grateful to the patients who participated in this study and to Kerry Guest and Richard McDonald, Watermeadow Medical, an Ashfield Company, part of UDG Healthcare plc. (supported by Novo Nordisk) for writing and editing assistance.

\section{Authors' Contributions}

All named authors met the International Committee of Medical Journal Editors (ICMJE) criteria for authorship for this article. S.K. was the lead study manager and therefore contributed to study design and conduct, and data acquisition. All authors had full access to all the study data and contributed to the interpretation, critically reviewed the article, and approved the final version for submission.

\section{Author Disclosure Statement}

T.D. has received research support or has consulted for Abbott, AstraZeneca, Bayer, Boehringer, DexCom, Insulet Corp., Eli Lilly, Medtronic, NovoNordisk, Roche, and is a shareholder of DreaMed Ltd. M.A.S., S.K., Y.K., and T.W. are employees of Novo Nordisk and own shares in Novo Nordisk. R.Z. has consulted for Abbott, AstraZeneca, BerlinChemie, DexCom, Novo Nordisk, and Roche Diabetes Care.

\section{Funding Information}

This study was sponsored by Novo Nordisk. Medical writing and editing support were also funded by Novo Nordisk.

\section{Supplementary Material}

Supplementary Figure S1

Supplementary Figure S2

Supplementary Figure S3

Supplementary Figure S4

Supplementary Table S1

Supplementary Table S2

Supplementary Table S3

\section{References}

1. American Diabetes Association: 9. Pharmacologic approaches to glycemic treatment: Standards of Medical Care in Diabetes-2020. Diabetes Care 2020;43(Suppl 1):S98S110.
2. Russell-Jones D, Bode BW, De Block C, et al.: Fast-acting insulin aspart improves glycemic control in basal-bolus treatment for type 1 diabetes: results of a 26-week multicenter, active-controlled, treat-to-target, randomized, parallelgroup trial (onset 1). Diabetes Care 2017;40:943-950.

3. Mathieu C, Bode BW, Franek E, et al.: Efficacy and safety of fast-acting insulin aspart in comparison with insulin aspart in type 1 diabetes (onset 1): a 52-week, randomized, treat-to-target, phase III trial. Diabetes Obes Metab 2018; 20:1148-1155.

4. Buse JB, Carlson AL, Komatsu M, et al.: Fast-acting insulin aspart versus insulin aspart in the setting of insulin degludec-treated type 1 diabetes: efficacy and safety from a randomized double-blind trial. Diabetes Obes Metab 2018; 20:2885-2893.

5. Klonoff DC, Evans ML, Lane W, et al.: A randomized, multicentre trial evaluating the efficacy and safety of fastacting insulin aspart in continuous subcutaneous insulin infusion in adults with type 1 diabetes (onset 5). Diabetes Obes Metab 2019;21:961-967.

6. Kildegaard J, Buckley ST, Nielsen RH, et al.: Elucidating the mechanism of absorption of fast-acting insulin aspart: the role of niacinamide. Pharm Res 2019;36:49.

7. Heise T, Pieber TR, Danne T, et al.: A pooled analysis of clinical pharmacology trials investigating the pharmacokinetic and pharmacodynamic characteristics of fast-acting insulin aspart in adults with type 1 diabetes. Clin Pharmacokinet 2017;56:551-559.

8. Bowering K, Case C, Harvey J, et al.: Faster aspart versus insulin aspart as part of a basal-bolus regimen in inadequately controlled type 2 diabetes: the onset 2 trial. Diabetes Care 2017;40:951-957.

9. Rodbard HW, Tripathy D, Vidrio Velazquez M, et al.: Adding fast-acting insulin aspart to basal insulin significantly improved glycaemic control in patients with type 2 diabetes: a randomized, 18-week, open-label, phase 3 trial (onset 3). Diabetes Obes Metab 2017;19:1389-1396.

10. Lane WS, Favaro E, Rathor N, et al.: A randomized trial evaluating the efficacy and safety of fast-acting insulin aspart compared with insulin aspart, both in combination with insulin degludec with or without metformin, in adults with type 2 diabetes (onset 9). Diabetes Care 2020;43:1710-1716.

11. Bode BW, Iotova V, Kovarenko M, et al.: Efficacy and safety of fast-acting insulin aspart compared with insulin aspart, both in combination with insulin degludec, in children and adolescents with type 1 diabetes: the onset 7 Trial. Diabetes Care 2019;42:1255-1262.

12. Anderson JH, Jr, Brunelle RL, Koivisto VA, et al.: Reduction of postprandial hyperglycemia and frequency of hypoglycemia in IDDM patients on insulin-analog treatment. Multicenter Insulin Lispro Study Group. Diabetes 1997;46:265-270.

13. Home PD, Lindholm A, Hylleberg B, et al.: Improved glycemic control with insulin aspart: a multicenter randomized double-blind crossover trial in type 1 diabetic patients. UK Insulin Aspart Study Group. Diabetes Care 1998;21:1904-1909.

14. Andersen G, Meiffren G, Lamers D, et al.: Ultra-rapid BioChaperone Lispro improves postprandial blood glucose excursions vs insulin lispro in a 14-day crossover treatment study in people with type 1 diabetes. Diabetes Obes Metab 2018;20:2627-2632.

15. Battelino T, Danne T, Bergenstal RM, et al.: Clinical targets for continuous glucose monitoring data interpretation: 
recommendations from the International Consensus on Time in Range. Diabetes Care 2019;42:1593-1603.

16. Bailey T, Bode BW, Christiansen MP, et al.: The performance and usability of a factory-calibrated flash glucose monitoring system. Diabetes Technol Ther 2015;17:787794.

17. Evans M, Welsh Z, Ells S, et al.: The impact of flash glucose monitoring on glycaemic control as measured by HbA1c: a meta-analysis of Clinical Trials and Real-World Observational Studies. Diabetes Ther 2020;11:83-95.

18. Guidelines for Good Pharmacoepidemiology Practice (GPP). Pharmacoepidemiol Drug Saf 2016;25:2-10.

19. World Medical Association: World Medical Association Declaration of Helsinki: ethical principles for medical research involving human subjects. JAMA 2013;310:21912194.

20. Danne T, Nimri R, Battelino T, et al.: International consensus on use of continuous glucose monitoring. Diabetes Care 2017;40:1631-1640.

21. Treatment Related Impact Measure-Diabetes (TRIM-D) User Manual_Version 1.1; August 2017. https://eprovide.mapitrust.org/instruments/treatment-related-impact-measure-fordiabetes

22. Novo Nordisk: TRIM-D questionnaire-TRIM-D ${ }^{\odot}$ version of 04 Nov 08. German (Germany): MAPI Research Institute, 2008

23. Bradley C: Diabetes Treatment Satisfaction Questionnaire (DTSQ) User Guidelines: Health Psychology Research Ltd., 2016.

24. C B. DTSQc ${ }^{\circledR}$. German for Germany 19.5 .06 (from standard UK English rev. 4.3.98; Intro. for Novo Nordisk 24.3.06), 2006.

25. Runge AS, Kennedy L, Brown AS, et al.: Does time-inrange matter? Perspectives from people with diabetes on the success of current therapies and the drivers of improved outcomes. Clin Diabetes 2018;36:112-119.

26. Gabbay MAL, Rodacki M, Calliari LE, et al.: Time in range: a new parameter to evaluate blood glucose control in patients with diabetes. Diabetol Metab Syndr 2020;12:22.

27. Advani A: Positioning time in range in diabetes management. Diabetologia 2020;63:242-252.

28. Guo Q, Zang $\mathrm{P}, \mathrm{Xu} \mathrm{S}$, et al.: Time in range, as a novel metric of glycemic control, is reversely associated with presence of diabetic cardiovascular autonomic neuropathy independent of $\mathrm{HbA} 1 \mathrm{c}$ in Chinese type 2 diabetes. J Diabetes Res 2020;2020:5817074.

29. Beck RW, Bergenstal RM, Riddlesworth TD, et al.: Validation of time in range as an outcome measure for diabetes clinical trials. Diabetes Care 2019;42:400-405.
30. Mayeda L, Katz R, Ahmad I, et al.: Glucose time in range and peripheral neuropathy in type 2 diabetes mellitus and chronic kidney disease. BMJ Open Diabetes Res Care 2020;8:e000991.

31. Lu J, Ma X, Zhou J, et al.: Association of time in range, as assessed by continuous glucose monitoring, with diabetic retinopathy in type 2 diabetes. Diabetes Care 2018;41: 2370-2376.

32. Service FJ: Glucose variability. Diabetes 2013;62:13981404.

33. Sartore G, Chilelli NC, Burlina S, et al.: Association between glucose variability as assessed by continuous glucose monitoring (CGM) and diabetic retinopathy in type 1 and type 2 diabetes. Acta Diabetol 2013;50:437-442.

34. Soupal J, Skrha J, Jr., Fajmon M, et al.: Glycemic variability is higher in type 1 diabetes patients with microvascular complications irrespective of glycemic control. Diabetes Technol Ther 2014;16:198-203.

35. Agiostratidou G, Anhalt H, Ball D, et al.: Standardizing clinically meaningful outcome measures beyond $\mathrm{HbA} 1 \mathrm{c}$ for type 1 diabetes: a consensus report of the American Association of Clinical Endocrinologists, the American Association of Diabetes Educators, the American Diabetes Association, the Endocrine Society, JDRF International, The Leona M. and Harry B. Helmsley Charitable Trust, the Pediatric Endocrine Society, and the T1D Exchange. Diabetes Care 2017;40:1622-1630.

36. Blonde L, Khunti K, Harris SB, et al.: Interpretation and impact of real-world clinical data for the practicing clinician. Adv Ther 2018;35:1763-1774.

37. Petersmann: Diabetologie 2019;14:S111-S118.

38. German Medical Association: Neufassung der Richtlinie der Bundesärztekammer zur Qualitätssicherung laboratoriumsmedizinischer Untersuchungen-Rili-BÄK [New version of the guideline of the German Medical Association for quality assurance of medical laboratory examinations (RiliBÄK)]. Dtsch Arztebl 2019;116:A-2422/B-1990/C-1930.

Address correspondence to: Thomas Danne, MD

Diabetes Center for Children and Adolescents Kinder- und Jugendkrankenhaus AUF DER BULT

Hanover Medical School Janusz-Korczak-Allee 12

Hannover 30173

Germany

E-mail: danne@hka.de 